\title{
Outcome Study of Anterior Cervical Discectomy and Fusion with Lordotic Cage Insertion
}

\author{
Nattawut Niljianskul, M.D. \\ Division of Neurosurgery, Department of Surgery, Faculty of Medicine Vajira Hospital, Navamindradhiraj University, \\ Dusit, Bangkok 10300, Thailand. \\ Received 14 December 2020 • Revised 11 February 2021 • Accepted 11 February 2021 • Published online 30 April 2021
}

\begin{abstract}
:
Objective: This study retrospectively evaluated the clinical and radiographic outcomes following the use of a lordotic cage in anterior cervical discectomy and fusion (ACDF).
\end{abstract}

Material and Methods: All patients who underwent ACDF, at Vajira Hospital; between May 2017 and May 2020, were included in this study. Radiographic images were used to evaluate the device-level Cobb angle (DLCA), segmental Cobb angle (SCA), global Cobb angle (GCA), sagittal vertical axis (SVA), sagittal alignment (SA), and intervertebral disk height. The visual analog scale (VAS) for neck pain, and the Japanese Orthopaedic Association (JOA) score were reviewed as part of the patient's medical records. Preoperative DLCA, SCA, GCA, SVA, SA, and intervertebral disk height measurements were compared with postoperative measurements at 1 year.

Results: A total of 51 patients (88 disks), having undergone ACDF with lordotic cage insertion were included in this study. The initial curvature of the cervical spine was diagnosed as kyphosis in 30 (58.8\%) patients, and as lordosis in 21 $(41.2 \%)$ patients. There was significant improvement in the VAS, JOA, DLCA, SCA, GCA, SVA, SA, and intervertebral disk height after ACDF ( $p$-value<0.050). In patients with preoperative kyphosis, the greatest changes were observed in the GCA (p-value=0.004).

Conclusion: The use of a lordotic cage in ACDF improved both the clinical and radiographic outcomes of all postoperative parameters, regardless of the patient's preoperative cervical spine curvature; although, patients with preoperative kyphosis had greater improvement in GCA.

Keywords: alignment, anterior cervical discectomy and fusion, lordotic cage

Contact: Nattawut Niljianskul, M.D.

Division of Neurosurgery, Department of Surgery, Faculty of Medicine Vajira Hospital, Navamindradhiraj University, Dusit, Bangkok 10300, Thailand.

E-mail: nattawut@nmu.ac.th

This is an open access article under the CC BY-NC-ND license

(http://www.jhsmr.org/index.php/jhsmr/about/editorialPolicies\#openAccessPolicy).

J Health Sci Med Res 2022;40(1):11-17 doi: 10.31584/jhsmr.2021808 www.jhsmr.org 


\section{Introduction}

Anterior cervical discectomy and fusion (ACDF) is a common operation for neural decompression in degenerative disk disease, and cervical spondylosis with radiculopathy or myelopathy. Although, the rate of fusion from autologous bone grafts is higher than with allografts, autologous bone grafts obtained from the anterior iliac crest are associated with significant donor site morbidity and complications; including, chronic pain, hematomas, infections, iliac crest fractures, and unsightly scars. ${ }^{1}$ Cervical interbody cages, including polyetheretherketone (PEEK) cages, titanium cages, and carbon fiber cages, have been used to prevent these complications.

The characteristics of PEEK make it a favorable option for ACDF, because it comprises of a semicrystalline polyaromatic linear polymer with elasticity properties that closely match those of cortical bone. Chou et al. ${ }^{2}$ retrospectively compared the results of anterior cervical fusion using titanium cages, PEEK cages, and tricortical bone grafts. They documented better fusion rates and less subsidence when PEEK cages were used, because PEEK has greater elasticity than titanium.

The normal anatomic alignment of the cervical spine is lordosis. In animal model studies, kyphosis alone, and without cord compression, increased longitudinal cord tension and intramedullary pressure; resulting in neuronal loss and demyelination. ${ }^{3,4}$ Villavicencio et al. ${ }^{5}$ reported that non-lordotic alignment patients had increased, degenerative change in the cervical spine within 10 years. Uchida et al. ${ }^{6}$ determined that among patients with cervical spondylotic myelopathy (CSM) who had kyphotic deformity, correction of sagittal alignment (SA) of the vertebrae significantly improved neurologic outcome. The authors noted that a kyphotic alignment may contribute to cervical myelopathy, due to longitudinal distraction, resulting in a pathophysiology that is the same as tethered cord syndrome. The loss of lordosis and the development of a kyphosis can be associated with neurologic deterioration, and should be avoided. ${ }^{7}$ Koeppen et al. reported on 102 patients diagnosed with CSM, representing 219 fused levels treated with a lordotic PEEK cage. They reported that kyphosis is associated with preoperative and postoperative neck pain. The goal of ACDF is decompression of neural structures, stability, and restoration of lordosis. ${ }^{8}$

At present, PEEK cages can be categorized into three types: standard cages (no lordotic angle), lordotic cages, and convex cages. Most ACDFs that are performed globally use various type of cages. It is not known whether the use of lordotic cages can increase device-level lordosis or intervertebral disk height only. Aligning the cervical spine with the lordosis results in a better patient outcome. The objective of this study was to examine the efficacy of lordotic PEEK cages used in ACDF, by assessing clinical outcomes and cervical spine alignment 1 year after surgery.

\section{Material and Methods}

The Ethics Committee of Vajira Hospital approved this study (087/63). All patients who underwent ACDF, at the neurosurgical department of Vajira hospital; between May 2017 and May 2020, and met certain exclusion criteria were included in this study. The indications for ACDF were trauma, spondylosis, ossification of posterior longitudinal ligament, or tumors. Patients whose records did not contain an operative note about the type of cage used, complete or relevant preoperative or postoperative imaging, or complete follow-up data were excluded. A total of 51 patients (88 disks) who underwent ACDF with lordotic cage insertion were included in this study.

Patient records were reviewed with preoperative visual analogue scale (VAS), the Japanese Orthopaedic Association (JOA) score, $\mathrm{X}$-rays of the cervical spine, and $\mathrm{MRI}$ of the spine being assessed. The procedures were performed using general anesthesia, but without neuromuscular blockers or agents that affect neuromuscular 
monitoring. Intraoperative neuromuscular monitoring was perofrmed to evaluate somatosensory-evoked potentials, transcranial motor-evoked potentials, and electromyography (EMG). Train-of-four monitoring twitches was conducted at the common peroneal nerve. A response rate of $75.0 \%$, or more was required before the EMG was recorded.

An anterior cervical approach was performed using a Smith-Robinson technique in all cases, by two experienced spine neurosurgeons. A cervical retractor was applied for distraction, followed by discectomy, removal of osteophytes by a high-speed drill and Kerrison rongeurs, and opening of the posterior longitudinal ligament under a microscope. After the fusion bed was prepared, interbody fusion was performed using lordotic PEEK cages that were $15 \mathrm{~cm}$ wide and $13 \mathrm{~cm}$ long, with a $5^{\circ}$ lordotic taper. The cages were filled with bone graft substitute. The third-generation cervical plates were inserted, and secured with variable angle screws in all patients. These plates were made from titanium and had an integral locking mechanism that avoids screw pullout. A soft collar was applied for a period of 6 weeks after surgery in all patients.

All patients underwent physical examination and $\mathrm{X}$-ray imaging of the cervical spine at 1 year. The $\mathrm{X}$-rays were reviewed, by the operator and another neurosurgeon, and were used to establish postoperative SA measurements. Calculations were conducted using the device-level Cobb angle (DLCA) at each operative level, the segmental Cobb angle (SCA) at two or more levels, the global Cobb angle (GCA), the sagittal vertical axis (SVA), and the SA by Gore method. ${ }^{9}$ Preoperative and postoperative SA measurements were compared. The extent of height reduction in the ventral and dorsal segments was also measured at 1 year follow-up, and preoperative and postoperative findings were compared. A reduction in height of more than $3 \mathrm{~cm}$ of either the ventral or dorsal segment was defined as subsidence. ${ }^{10}$ Operative time, blood loss, and complications were recorded. Dysphagia was evaluated by using the technique described by Bazaz. ${ }^{11}$

Statistical analysis was performed using the Statistical Package for the Social Sciences software version 22 for Windows (SPSS Inc., Chicago, IL, the USA). The t-test was used to analyze continuous quantitative variables. Preoperative and postoperative cervical parameters were compared by ANOVA, and the p-values were calculated. A $p$-value of <.050 was considered statistically significant.

\section{Results}

The demographic data for the patients in this study are presented in Table 1. Initially, 54 patients were included in the study; however, one patient was eventually excluded because the type of cage used was not recorded in the patient's operative notes, and two patients were excluded because of incomplete post-operative imaging. The 51 patients included in the study represented 88 fused intervertebral disks. There were 28 males and 23 females, between 25 and 78 years of age, with a median age of 57 years. The most common indications for ACDF were spondylosis (54.9\%) and trauma (35.5\%). In 30 patients $(58.8 \%)$, the preoperative curvature of the cervical spine was kyphosis, and in 21 patients (41.2\%) it was lordosis. Most patients (52.9\%) underwent single-level ACDF, and the most common fused level was C5-6 (42.0\%). The cohort did not present with postoperative neurologic deficits, infection, or hematoma. Postoperative dysphagia was present in two patients (3.9\%); hoarseness in two (3.9\%); and subsidence of the cage in seven (13.7\%).

The mean changes for patients who underwent ACDF with lordotic PEEK cages were assessed and documented across the cohort. The mean VAS for neck pain across the cohort was $5.5( \pm 1.8)$ preoperatively and $5.2( \pm 0.8)$ postoperatively, improving by a mean of 0.2 $( \pm 0.7)$; which was considered statistically significant. The 
Table 1 Patient and treatment characteristic

\begin{tabular}{ll}
\hline Characteristic & All $(\mathbf{n = 5 1 )}$ \\
\hline Sex & \\
Male & $28(54.9)$ \\
Female & $23(45.1)$ \\
Age, years & $57(25.0-78.0)$ \\
Diagnosis & \\
Trauma & $18(35.5)$ \\
Spondylosis & $28(54.9)$ \\
Tumor & $1(2.0)$ \\
OPLL & $4(7.8)$ \\
Curvature of cervical spine & \\
Lordosis & $21(41.2)$ \\
Kyphosis & $30(58.8)$ \\
Operation time. minutes & $173(100.0-380.0)$ \\
Blood loss, milliliter & $50(10.0-1,400.0)$ \\
Length of stay, days & $7(4.0-51.0)$ \\
Fused level & \\
C3/4 & $9(10.2)$ \\
C4/5 & $22(25.0)$ \\
C5/6 & $37(42.0)$ \\
C6/7 & $20(22.7)$ \\
Number of fused levels & \\
1 & $27(52.9)$ \\
2 & $15(29.4)$ \\
3 & $6(11.8)$ \\
4 & $3(5.9)$ \\
Post-operative complications & $2(3.9)$ \\
Dysphagia & $2(3.9)$ \\
Hoarseness & $7(13.7)$ \\
Subsidence & $39(76.5)$ \\
None &
\end{tabular}

Values are presented as number (\%) or median (range)

OPLL=ossification of posterior longitudinal ligament mean JOA score was $13.0( \pm 4.3)$ preoperatively and 15.2 ( \pm 4.0 ) postoperatively, improving by a mean of $2.2( \pm 1.2)$; which was considered statistically significant. The mean parameters; including, DLCA, SCA, GCA, C2-7 SVA, and SA, were improving toward lordosis after surgery; which were considered statistically significant. The mean fused level intervertebral disk height was $4.4 \mathrm{~cm} \quad( \pm 1.9$ $\mathrm{cm})$ preoperatively and $5 \mathrm{~cm}( \pm 2 \mathrm{~cm})$ at 1 year follow-up, demonstrating a significant increase between preoperative and postoperative measurements (Table 2).

The results were analyzed based on preoperative alignment, in which 21 patients had cervical lordosis and 30 had cervical kyphosis. In patients with cervical kyphosis, the improvement in GCA toward lordosis was statistically significant (Table 3). In Table 4, the changes in SA and fused level intervertebral disk height were compared between patients older than 60 years and those 60 years of age and younger. There were no statistically significant differences between the two age groups in terms of cervical alignment parameters, or fused level intervertebral disk height for patients undergoing ACDF with lordotic PEEK cages.

Table 2 Comparison of clinical and radiographic features

\begin{tabular}{|c|c|c|c|c|}
\hline Variables & Pre-operative & Post-operative & Change & $p$-value \\
\hline VAS neck & $5.5(1.8)$ & $0.2(0.7)$ & $5.2(1.8)$ & $<0.001$ \\
\hline JOA score & $13.0(4.3)$ & $15.2(4.0)$ & $2.2(1.2)$ & $<0.001$ \\
\hline DLCA, degree & $4.5(3.8)$ & $8.1(4.8)$ & $3.6(3.9)$ & $<0.001$ \\
\hline SCA, degree & $6.7(4.1)$ & $11.1(5.3)$ & $4.4(4.8)$ & $<0.001$ \\
\hline GCA, degree & $13.6(8.7)$ & $18.8(7.5)$ & $5.2(7.7)$ & $<0.001$ \\
\hline C2-7 SVA, cm & $1.7(0.9)$ & $2.2(0.8)$ & $4.9(1.0)$ & 0.001 \\
\hline Sagittal alignment, degree & $12.9(7.2)$ & $18.9(7.3)$ & $6.0(5.9)$ & $<0.001$ \\
\hline Intervertebral disc height, $\mathrm{cm}$ & $4.4(1.9)$ & $5.0(2.0)$ & $0.6(0.7)$ & $<0.001$ \\
\hline
\end{tabular}

Values are presented as mean (S.D.)

VAS=visual analogue scale for neck pain, JOA=the Japanese Orthopaedic Association score, DLCA=device level Cobb angle, SCA=segmental Cobb angle, GCA=global Cobb angle, SVA=sagittal vertical axis, $\mathrm{cm}=$ centimeter 
Table 3 Subgroup analysis by pre-operative alignment

\begin{tabular}{|c|c|c|c|c|c|}
\hline \multirow{2}{*}{ Variables } & \multicolumn{2}{|c|}{ Initial Iordosis } & \multicolumn{2}{|c|}{ Initial kyphosis } & \multirow{2}{*}{$p$-value } \\
\hline & Mean (S.D.) & No. & Mean (S.D.) & No. & \\
\hline$\triangle \mathrm{DLCA}$, degree & $4(4.6)$ & 34 & $3.4(3.4)$ & 54 & 0.480 \\
\hline$\triangle \mathrm{SCA}$, degree & $4.7(3.7)$ & 21 & $4.2(5.5)$ & 30 & 0.730 \\
\hline$\triangle \mathrm{GCA}$, degree & $1.6(5.1)$ & 21 & $7.7(8.2)$ & 30 & 0.004 \\
\hline$\triangle \mathrm{C} 2-7 \mathrm{SVA}, \mathrm{cm}$ & $0.6(0.8)$ & 21 & $0.4(1.1)$ & 30 & 0.550 \\
\hline$\Delta$ Sagittal alignment, degree & $4.7(5.3)$ & 21 & $6.9(5.9)$ & 30 & 0.170 \\
\hline$\Delta$ Intervertebral disc height, $\mathrm{cm}$ & $0.6(0.7)$ & 21 & $0.6(0.7)$ & 30 & 0.820 \\
\hline
\end{tabular}

Values are presented as mean (S.D.)

Abbreviations: $\triangle=$ change in, DLCA=device level Cobb angle, SCA=segmental Cobb angle, GCA=global Cobb angle, SVA=sagittal vertical axis, $\mathrm{cm}=$ centimeter

Table 4 Subgroup analysis by age

\begin{tabular}{|c|c|c|c|c|c|}
\hline \multirow{2}{*}{ Variables } & \multicolumn{2}{|c|}{ Age $\leq 60$ year } & \multicolumn{2}{|c|}{ Age $>60$ year } & \multirow{2}{*}{$p$-value } \\
\hline & Means (S.D.) & No. & Means (S.D.) & No. & \\
\hline$\triangle \mathrm{DLCA}$, degree & $3.8(4.3)$ & 56 & $3.4(3.1)$ & 32 & 0.650 \\
\hline$\triangle \mathrm{SCA}$, degree & $4.3(4.2)$ & 35 & $4.8(5.9)$ & 16 & 0.690 \\
\hline$\triangle \mathrm{GCA}$, degree & $5.0(7.5)$ & 35 & $5.7(8.2)$ & 16 & 0.780 \\
\hline$\triangle \mathrm{C} 2-7 \mathrm{SVA}, \mathrm{cm}$ & $0.5(1.1)$ & 35 & $0.5(0.7)$ & 16 & 0.890 \\
\hline$\Delta$ Sagittal alignment, degree & $5.5(5.3)$ & 35 & $7.0(6.4)$ & 16 & 0.380 \\
\hline$\Delta$ Intervertebral disc height, $\mathrm{cm}$ & $0.6(0.7)$ & 35 & $0.6(0.7)$ & 16 & 1.000 \\
\hline
\end{tabular}

Values are presented as mean (S.D.)

Abbreviations: $\Delta=$ change in, DLCA=device level Cobb angle, SCA=segmental Cobb angle, GCA=global Cobb angle, SVA=sagittal vertical axis, $\mathrm{cm}=$ centimeter

\section{Discussion}

Cervical spine alignment is an important factor for clinical outcomes in many studies. ${ }^{12,13}$ This study assessed the clinical outcome and the cervical spine alignment in patients 1 year after undergoing ACDF with a lordotic PEEK cage. It found that ACDF with lordotic PEEK cages not only improved VAS for neck pain and JOA score, but also demonstrated a statistically significant restoration of cervical lordosis alignment. In patients with preoperative cervical kyphosis, the use of a lordotic PEEK cage significantly increased the GCA. This suggests that patients who have cervical kyphosis are likely to benefit from the use of lordotic
PEEK cages during ACDF. For some of the patients, who had preoperative cervical kyphosis, their cervical alignment had improved to lordosis after surgery. The changes in cervical curvature may be due to use of a lordotic PEEK cage, or reduced symptoms of neck pain from surgery; as can be seen from improvement in VAS compared to preoperative and postoperative. This allows the necks of these patients to become restored lordosis. The GCA, after surgery in patients with preoperative cervical kyphosis; were therefore more improved than in the other groups.

Kashani et al. ${ }^{14}$ demonstrated that ACDF is more effective in improving disability and reducing pain in patients 
older than 45 years of age, although the findings regarding pain were not statistically significant. Chotai et al. ${ }^{15}$ reported that patients older than 65 years of age have a slightly higher cost utility ratio compared to the younger patient group. Additionally, surgery in the older cohort did provide a significant improvement in pain, disability, and qualityof-life outcomes. This current study found no age-related differences in the cervical alignment parameters, or the fused level intervertebral disk height with lordotic PEEK cages in ACDF. This would indicate that patients can benefit from a lordotic PEEK cage regardless of age.

Dysphagia is a common complication of ACDF, occurring in between $2.0 \%$ and $67.0 \%$ of patients in the early postoperative period. ${ }^{11}$ Most of these symptoms disappear within the first 3 months after surgery. The incidence of chronic dysphagia after ACDF is between $3.0 \%$ and $21.0 \%{ }^{16}$ In this cohort, 10 patients (19.6\%) reported dysphagia in the immediate postoperative period, which resolved after 6 months in eight patients (15.7\%). Only two patients (3.9\%) continued to complain about chronic dysphagia 1 year after surgery. The pathologic mechanism of postoperative dysphagia is still unknown, but it may be associated with implant-related mechanical issues, intraoperative esophageal retraction, and adhesions. ${ }^{17}$ All of the patients in this cohort, who reported chronic dysphagia, had operative times of more than 4 hours, and a cervical plate and screws were used for fixation during ACDF. The author considers that these symptoms may be from prolonged esophageal retraction during surgery, or may be related to the cervical plate implant.

Subsidence was a common postoperative problem that could lead to deterioration of long-term function. It will cause loss of correction of the segmental angle and the Cobb angle from C2-7. Loss of cervical lordosis is a risk factor to degeneration in the adjacent levels. ${ }^{18}$ Criteria for evaluating subsidence are still lacking. The most common method is to measure the postoperative reduction in the heights of the ventral and dorsal segments. Subsidence of less than $2 \mathrm{~cm}$ is acceptable. ${ }^{19}$

The incidence of cage subsidence with the PEEK cages ranged from $0.0 \%$ to $18.0 \%$ in many studies ${ }^{7,20}$, which is consistent with the $17.0 \%$ PEEK cage subsidence rate reported in this current study. In many studies, the rate of subsidence in patients not using a postoperative external collar was reported to be high. This led to the decision to prescribe a soft collar for 6 weeks after surgery for all patients. ${ }^{21,22}$ Although, a $17.0 \%$ subsidence rate was reported, it did not cause any postoperative neurologic deficit or revision surgery in 1-year follow-up.

This current study has several limitations. The author did not compare the lordotic PEEK cage with other types of cages. Moreover, only a small number of patients were included. Finally, this is a retrospective study.

\section{Conclusion}

The use of the lordotic PEEK cage in ACDF improved both clinical and radiographic outcomes in corrected cervical spine alignment. Preoperative cervical kyphosis patients initially have greater GCA improvement than that of lordosis patients.

\section{Conflict of interest}

None

\section{References}

1. Samartzis D, Shen F, Goldberg EJ, An HS. Is autograft the gold standard in achieving radiographic fusion with rigid anterior plate fixation?. Spine 2005;30:1756-61

2. Chou YC, Chen DC, Hsieh WA, Chen WF, Yen PS, Harnod T, et al. Efficacy of anterior cervical fusion: comparison of titanium cages, polyetheretherketone (PEEK) cages and autogenous bone grafts. J Clin Neurosci 2008;15:1240-5.

3. Farley CW, Curt BA, Pettigrew DB, Holtz JR, Dollin N, Kuntz C $4^{\text {th }}$. Spinal cord intramedullary pressure in thoracic kyphotic deformity: a cadaveric study. Spine (Phila Pa 1976) 2012;37: E224-30 
4. Shimizu K, Nakamura M, Nishikawa Y, Hijikata S, Chiba K, Toyama Y. Spinal kyphosis causes demyelination and neuronal loss in the spinal cord: a new model of kyphotic deformity using juvenile Japanese small game fowls. Spine (Phila Pa 1976) 2005;30:2388-92.

5. Villavicencio AT, Babuska JM, Ashton A, Busch E, Roeca C, Nelson EL, et al. Prospective, randomized, double-blind clinical study evaluating the correlation of clinical outcomes and cervical sagittal alignment. Neurosurgery 2011;68:1309-16.

6. Uchida K, Nakajima H, Sato R, Yayama T, Mwaka ES, Kobayashi S, et al. Cervical spondylotic myelopathy associated with kyphosis or sagittal sigmoid alignment: outcome after anterior or posterior decompression. J Neurosurg 2009;11: 521-8.

7. Cabraja M, Abbushi A, Kroppenstedt S, Woiciechowsky C. Cages with fixation wings versus cages plus plating for cervical reconstruction after corpectomy-is there any difference?. Cent Eur Neurosurg 2010;71:59-63.

8. Koeppen D, Piepenbrock C, Kroppenstedt S, Čabraja M. The influence of sagittal profile alteration and final lordosis on the clinical outcome of cervical spondylotic myelopathy. A Delta-Omega-analysis. PLoS One 2017;12:e0174527.

9. Gore DR. Roentgenographic findings in the cervical spine in asymptomatic persons: a ten-year follow-up. Spine 2001;26: 2463-6.

10. Gercek E, Arlet V, Delisle J, Marchesi D. Subsidence of standalone cervical cages in anterior interbody fusion: warning. Eur Spine J 2003;12:513-6.

11. Bazaz R, Lee MJ, Yoo JU. Incidence of dysphagia after anterior cervical spine surgery: a prospective study. Spine (Phila Pa 1976) 2002;27:2453-8.

12. Bao H, Varghese J, Lafage R, Liabaud B, Diebo B, Ramchandran $\mathrm{S}$, et al. Principal radiographic characteristics for cervical spinal deformity: a health-related quality-of-life analysis. Spine (Phila Pa 1976) 2017;42:1375-82.

13. Steinmetz MP, Stewart TJ, Kager CD, Benzel EC, Vaccaro AR. Cervical deformity correction. Neurosurgery 2007;2007; 60(1 Suppl 1):S90-7.
14. Omidi KF, Ghayem HE, Ghandehari R. Impact of Age and Duration of Symptoms on Surgical Outcome of Single-Level Microscopic Anterior Cervical Discectomy and Fusion in the Patients with Cervical Spondylotic Radiculopathy. Neurosci J 2014;2014:808596.

15. Chotai S, Parker SL, Sielatycki JA, Sivaganesan A, Kay HF, Wick JB, et al. Impact of old age on patient-report outcomes and cost utility for anterior cervical discectomy and fusion surgery for degenerative spine disease. Eur Spine J 2017;26: 1236-45.

16. Riley LH, Skolasky RL, Albert TJ, Vaccaro AR, Heller JG. Dysphagia after anterior cervical decompression and fusion: Prevalence and risk factors from a longitudinal cohort study. Spine (Phila Pa 1976) 2005;30:2564-9.

17. Fountas KN, Kapsalaki EZ, Nikolakakos LG, Smisson HF, Johnston KW, Grigorian AA,et al. Anterior cervical discectomy and fusion associated complications. Spine (Phila Pa 1976) 2007;32:2310-7.

18. Katsuura A, Hukuda S, Saruhashi Y, Mori K. Kyphotic malalignment after anterior cervical fusion is one of the factors promoting the degenerative process in adjacent intervertebral levels. Eur Spine J 2001;10:320-4.

19. Gercek E, Arlet V, Delisle J, Marchesi D. Subsidence of standalone cervical cages in anterior interbody fusion: warning. Eur Spine J 2003;12:513-6.

20. Cabraja M, Oezdemir S, Koeppen D, Kroppenstedt S. Anterior cervical discectomy and fusion: comparison of titanium and polyetheretherketone cages. BMC Musculoskelet Disord 2012;13:172.

21. Schmieder K, Wolzik-Grossmann M, Pechlivanis I, Engelhardt M, Scholz M, Harders A. Subsidence of the wing titanium cage after anterior cervical interbody fusion: 2-year follow-up study. J Neurosurg Spine 2006;4:447-53.

22. Barsa $P$, Suchomel P. Factors affecting sagittal malalignment due to cage subsidence in standalone cage assisted anterior cervical fusion. Eur Spine J 2007;16:1395-400. 